\title{
L'INTERCOMPRÉHENSION POUR LA PROMOTION DE LA LANGUE FRANÇAISE DANS L'INTERNATIONALISATION DES UNIVERSITÉS BRÉSILIENNES
}

\section{INTERCOMPREHENSION FOR THE PROMOTION OF FRENCH LANGUAGE IN THE INTERNATIONALIZATION OF BRAZILIAN UNIVERSITIES}

Felipe Furtado Guimarães*, **

\begin{abstract}
RÉSUMÉ
Cette étude vise à examiner l'approche d'intercompréhension (AI) comme une occasion de promouvoir la langue française dans le contexte de l'internationalisation des universités brésiliennes, compte tenu des réformes récentes en matière d'éducation (en général) et d'enseignement des langues (en particulier) qui entravent l'enseignement, l'apprentissage et l'utilisation de langues autres que l'anglais au Brésil. À cette fin, l'auteur a effectué une recherche bibliographique pour identifier les rapports entre les études de langues étrangères et internationalisation, ainsi qu'une recherche documentaire pour identifier les textes relatifs aux réformes éducatives et politiques linguistiques au Brésil qui affectent l'utilisation du français. Il est conclu que des approches innovantes telles que l'AI sont nécessaires pour promouvoir l'enseignement du français (et d'autres langues) en milieu universitaire, avec le support des technologies de l'information et de la communication (TICs), en faveur d'un enseignement supérieur plus diversifié et multilingue.
\end{abstract}

Mots-Clés: intercompréhension; internationalisation; politiques linguistiques.

\section{ABSTRACT}

This study aimed at discussing the Intercomprehension Approach (IA) as an opportunity to promote the French language in the context of internationalization of Brazilian universities, considering recent reforms in education (in general) and in language teaching (in particular) which hinder the teaching, learning and use of languages which are not English, in Brazil. In order to achieve this goal, the author used bibliographic research to find studies on foreign languages and internationalization, as well as documental research to find texts related to educational reforms and language policies in Brazil, which influence the use of French. One concludes that innovative approaches are necessary, such as IA, to promote the teaching of French (and other languages) in university settings, with the support of information and communication technologies (ICTs), in order to promote a more diversified and multilingual higher education.

Keywords: intercomprehension; internationalization; language policies.

\section{INTRODUCTION}

L'internationalisation est un phénomène qui s'est développé dans le contexte de l'enseignement supérieur, sous l'impulsion du processus de mondialisation, qui a accru la mobilité des personnes, des biens, de l'information et des langues (WANG et al., 2014). La redéfinition des notions d'espace et de temps ainsi que l'extension de l'utilisation des technologies de l'information et de la communication (TICs) ont eu un impact sur la circulation de l'information, modifiant ainsi les relations des personnes avec les contextes et les réalités locaux et mondiaux (FINARDI et PORCINO, 2014). De plus, les changements apportés par la mondialisation et l'internationalisation ont créé un monde de super diversité (VERTOVEC, 2007; BLOMMAERT, 2013), avec des tensions entre valeurs et langues locales et mondiales (GUIMARÃES et al., 2019).

D'une part, la mondialisation tente de promouvoir l'intégration globale entre les sociétés, ce qui entraîne l'expansion de schémas culturels donnés. D'autre part, elle favorise également le processus d'individualisation et d'autonomie identitaire. En d'autres termes, les individus souhaitent (en même temps) s'intégrer dans le monde globalisé et ils souhaitent également que leur identité régionale / locale soit reconnue, y compris les langues qu'ils utilisent dans la vie quotidienne (DAY, 2012).

L'éducation (en général) et l'enseignement supérieur (en particulier) ont également été affectés par ces changements, qui sont devenus plus évidents récemment dans le processus d'internationalisation de l'enseignement

\footnotetext{
* Universidade Federal do Espírito Santo (UFES), Vitória, ES, Brésil.

** Universidad Pablo de Olavide (UPO) Espagne. felipeguim2@yahoo.com.br Orcid: https://orcid.org/0000-0001-6184-3691

Cette étude a été développée dans le cadre du Centre d'études en relations internationales de l'Université Fédérale d'Espirito Santo (NERI/ Ufes).
} 
supérieur (GUIMARÃES et al., 2020). Laus (2012) considère l'internationalisation comme une «réalité non équivoque», car ce thème est souvent mentionné dans la planification stratégique institutionnelle, dans les rapports d'organisations internationales (commel'Unesco' et L'Organisation de coopération et de développement économiques - $\mathrm{OCDE}^{2}$ ) et dans les articles scientifiques dans le monde entier (ALTBACH et KNIGHT, 2007; CANAGARAJAH, 2013; HAMEL, 2013; STEIN et ANDREOTTI, 2017).

En effet, l'internationalisation et la mondialisation sont des phénomènes connexes, tel que le discute Knight (2003) : l'internationalisation change le monde de l'éducation; et la mondialisation change le monde de l'internationalisation. Selon Spolsky (2004), en raison de la centralité de la langue dans l'éducation (principalement la langue d'enseignement), les langues jouent un rôle clé dans l'internationalisation et la mondialisation, et certains auteurs discutent même de l'internationalisation linguistique de l'enseignement supérieur (RAMOS-GARCIA et VAZQUEZ, 2018).

Dans le scénario brésilien, le rôle des langues a été récemment affecté par les modifications de la législation sur l'éducation, comme la Loi des directives nationales pour l'éducation (LDB, en portugais) ${ }^{3}$ qui favorise l'utilisation de l'anglais (GUIMARÃES et KREMER, 2020). Et aussi des changements apportés par des politiques d'internationalisation de l'enseignement supérieur, comme le programme Science sans Frontières $\left(\mathrm{SsF}_{s}\right)^{4}$ et le programme Capes PrInt $(\mathrm{PCP})^{5}$, aussi bien que programmes de développement des compétences linguistiques, comme le Langues sans Frontières $(\mathrm{LsF})^{6}$. Ces thèmes seront abordés plus loin. Par conséquent, en raison des changements promus par ces programmes, cette étude cherche à trouver des moyens de promouvoir la langue française dans les universités brésiliennes.

\section{REVUE DE LA LITTÉRATURE}

\subsection{Internationalisation}

L'une des définitions les plus répandues de l'internationalisation a été présentée par Knight (2003) l'internationalisation est définie comme le processus d'intégration d'une dimension internationale, interculturelle ou globale dans l'objectif, les fonctions et l'offre de l'enseignement supérieur. Cette définition a été mise à jour:

Le processus intentionnel d'intégration d'une dimension internationale, interculturelle ou mondiale dans l'objectif, les fonctions et l'offre de l'enseignement supérieur, afin d'améliorer la qualité de l'enseignement et de la recherche pour tous les étudiants et le personnel, et d'apporter une contribution significative à la société. (DE WIT et al, 2015, p. 29, notre traduction) ${ }^{7}$.

L'internationalisation peut aussi être vue comme un processus plus complet, comme un engagement, confirmé par l'action, d'intégrer des perspectives internationales et comparatives dans les missions d'enseignement, de recherche et de service de l'enseignement supérieur (HUDZIK, 2011). Il est essentiel qu'il soit adopté par la direction de l'établissement, la gouvernance, le corps professoral, les étudiants et toutes les unités de soutien.

La mondialisation et l'internationalisation ont des effets positifs et négatifs qui affectent différemment les pays et les régions, apportant plus d'avantages aux pays d'Europe et aux États-Unis qu'aux pays d'Amérique Latine et des Caraïbes (MENEGHEL; DE CAMARGO; SPELLER, 2018), qui font partie des pays du Sud Global (DE SOUSA SANTOS, 2011). Certains des effets de la mondialisation sur l'éducation sont mis en évidence par l'internationalisation et la transnationalisation de l'enseignement supérieur. Bernheim (2008) distingue ces deux processus en associant le premier à une motivation académique, en faveur de la coopération internationale mettant l'accent sur les relations horizontales et de soutien, tandis que le processus de transnationalisation est lié à un agenda économique et à la vision de l'enseignement supérieur en tant que service ou produit.

1. Plus d'informations sur: http://uis.unesco.org/en/uis-student-flow

2. Plus d'informations sur: https://data.oecd.org/students/international-student-mobility.htm

3. Plus d'informations sur: http://www.planalto.gov.br/ccivil_03/leis/19394.htm

4. Plus d'informations sur: http://cienciasemfronteiras.gov.br/web/csf/o-programa

5. Plus d'informations sur: https://capes.gov.br/cooperacao-internacional/multinacional/programa-institucional-de-internacionalizacaocapes-print

6. Plus d'informations sur: http://isf.mec.gov.br/

7. Texte original: The intentional process of integrating an international, intercultural or global dimension into the purpose, functions and delivery of post-secondary education, in order to enhance the quality of education and research for all students and staff, and to make a meaningful contribution to society. 
Les motivations à l'internationalisation sont discutées par Altbach et Knight (2007) et comprennent (mais ne sont pas limitées à) : avantage commercial, acquisition de connaissances et de langues, enrichissement du curriculum avec un contenu international, etc. En effet, Finardi et Rojo (2015), analysant deux universités brésiliennes, une publique et l'autre privée, indiquent que les institutions peuvent avoir différentes raisons d'internationaliser. Alors que les institutions publiques peuvent avoir des intérêts académiques, les institutions privées ne semblent pas s'intéresser à l'internationalisation, car le marché local de l'éducation au Brésil est assez confortable pour ces établissements privés, en raison du grand nombre d'étudiants brésiliens inscrits dans des établissements privés par rapport aux étudiants étrangers ${ }^{8}$.

L'internationalisation étant un thème relativement récent (du moins au Brésil - LAUS, 2012), il est important de signaler certaines idées fausses et certains mythes qui l'entourent. Knight (2011a) indique les mythes suivants : a) les étudiants étrangers deviennent des agents d'internationalisation; b) la réputation internationale agit comme un indicateur de la qualité; c) les nombreux accords internationaux sont un bon indicateur de l'internationalisation; d) l'accréditation internationale est une bonne reconnaissance de la qualité de l'éducation; e) un profil mondial est une bonne stratégie pour attirer les étudiants internationaux.

De Wit (2011) présente quelques idées fausses liées à l'internationalisation: a) l'enseignement dans une institution internationalisée doit se faire en anglais; b) internationaliser signifie étudier ou rester à l'étranger; c) avoir beaucoup d'étudiants internationaux est une bonne preuve d'internationalisation; d) il n'est pas nécessaire de tester les compétences interculturelles; e) plus de partenariats, plus international. Le fait que de nombreuses institutions pensent que l'internationalisation ne devrait se faire que par la langue anglaise (idée fausse «a ») peut avoir de graves conséquences sur d'autres langues (JENKINS, 2015; JORDÃO, 2016; BRUTT-GRIFFLER, 2017).

L'internationalisation ne se limite pas à la mobilité (DE WIT, 2011), mais de nombreuses activités dans ce processus sont toujours liées aux échanges universitaires à l'étranger. Une des plus grandes initiatives au Brésil pour favoriser la mobilité universitaire a été le programme Science sans Frontières $(\mathrm{Ss} F)$ - ce programme a été mis en œuvre entre 2011 et 2016 pour offrir plus de 100000 bourses, principalement au premier cycle (de l'enseignement supérieur), à des étudiants en sciences, en technologies, en génie et en mathématiques (STEM, dans l'acronyme en anglais).

Cependant, les candidats au programme SsF ont été confrontés à de graves problèmes de maîtrise de la langue pour étudier à l'étranger. Les premiers candidats ont choisi le Portugal ou l'Espagne (comme pays de destination) en raison de la proximité des langues parlées dans ces pays et du portugais brésilien (ALTENHOFEN, 2013). La faible maîtrise des brésiliens des langues étrangères a été rapportée à cette époque par les médias ${ }^{9}$ et a mis en lumière un problème que les experts discutaient depuis longtemps, avant l'instauration du SsF.

A cette époque, le gouvernement a créé le programme Anglais sans Frontières (AsF, en 2012) pour offrir aux participants du SsF la possibilité de développer leurs compétences, via des cours en ligne, des cours en face à face et des tests d'aptitude. Plus tard, ce programme a été étendu aux Langues sans Frontières (LsF, en 2014) - il sera présenté dans les sections suivantes. On devrait remarquer que ces programmes (AsF et LsF) ont favorisé la création de politiques linguistiques et de plans d'internationalisation, comme l'a suggeré Guimarães, Finardi et Casotti (2019).

Il convient de noter que l'internationalisation a été affectée par la réduction du financement de l'enseignement supérieur (ALTBACH et KNIGHT, 2007; KNOBEL, 2012; MANÇOS et COELHO, 2017) - 'une de ses conséquences étant la suspension du programme Science sans Frontières en 2016 et Langues sans Frontières en $2019^{10}$. Dans ce scénario, les technologies de l'information et de la communication (TICs) peuvent être utilisées pour promouvoir « l'internationalisation chez soi » (Internationalization at Home - laH - en anglais), définie par Beelen et Jones $(2015, \text { p. } 69 \text {, notre traduction })^{11}$ comme:

[...] l'intégration volontaire des dimensions internationales et interculturelles dans les programmes d'études formels et informels, pour tous les étudiants dans des environnements d'apprentissage nationaux.

8. Les établissements d'enseignement publics sont entièrement gratuits et financés par l'État. Seuls les établissements privés peuvent recevoir des frais de scolarité.

9. Plus d'informations sur: https://oglobo.globo.com/economia/emprego/brasileiros-nao-sabem-falar-ingles-apenas-5-dominam-idioma-6239142

10. Ce programme sera modifié et soutenu par l'Association brésilienne des gestionnaires des universités fédérales (Andifes, en portugais), au lieu du Ministère de l'Éducation.

11. Texte original: [...] the purposeful integration of international and intercultural dimensions into the formal and informal curriculum for all students within domestic learning environments. 
Un exemple d'IaH utilisant des technologies est appelé l'Apprentissage international collaboratif en ligne (Collaborative Online International Learning - COIL - en anglais - initialement développé à l'Université d'État de New York - SUNY) ${ }^{12}$, qui peut être utilisé aussi pour enseigner / apprendre des langues, comme l'ont indique Hildeblando Júnior et Finardi (2018).

Selon Finardi (2019a), les TICs et l'approche COIL peuvent également être utilisées, dans le contexte de l'enseignement supérieur et conjointement avec l'approche de l'intercompréhension, pour élargir les langues d'accès et d'internationalisation de l'enseignement supérieur. Afin de réfléchir à cette possibilité, la prochaine section décrira brièvement cette approche en décrivant leurs affordances (les opportunités) d'utilisation dans ce contexte.

\subsection{Intercompréhension}

Doyé (2005) définit l'intercompréhension comme une forme de communication dans laquelle chaque personne utilise sa propre langue et comprend celle des autres. Pour l'expliquer d'une manière différente, l'intercompréhension peut être conçue comme la capacité de comprendre d'autres langues, sans que l'on ait étudié ces langues. Une autre explication de cette approche a été présentée par la Commission Européenne et se réfère à une relation entre les langues dans laquelle des locuteurs de langues différentes (mais apparentées) peuvent facilement se comprendre sans une étude intentionnelle ni un effort extraordinaire (EUROPEAN COMMISSION, 2012).

Plusieurs auteurs ont discuté de ce sujet récemment. Araújo e Sá et Simões (2015) discutent des possibilités, des limites et des recommandations pour l'intégration de l'intercompréhension dans les programmes d'internationalisation. Meissner (2010) indique que l'intercompréhension peut être considérée comme une approche efficace dans les pays qui utilisent des langues romanes. De Oliveira (2016) discute de l'intercompréhension des langues romanes et de leur utilisation dans les cours d'anglais. De Biase (2013) souligne l'importance de l'intercompréhension en tant que stratégie d'apprentissage des langues romanes.

Ces auteurs présentent une vision positive de l'intercompréhension, bien que certains défis doivent être surmontés pour réussir la mise en œuvre de cette approche. En général, l'intercompréhension est considéré comme une alternative importante pour l'utilisation d'autres langues (comme le français) dans les contextes où l'anglais est devenu la langue dominante, comme dans les universités. Par conséquent, l'intercompréhension peut être une approche utile pour promouvoir le multilinguisme, au lieu de l'anglicisation - et c'est un aspect important dans les pays où l'anglais n'est pas la première langue.

L'approche d'intercompréhension (AI) est considérée comme l'une des idées les plus stimulantes pour la réalisation d'une éducation plurilingue - c'est une approche assez nouvelle, issue des discussions sur la didactique de l'enseignement/apprentissage des langues étrangères au début des années 90. L'exclusion de l'usage productif (habiletés d'expression orale et écrite) d'une langue étrangère est pertinente, car l'intercompréhension se caractérise par le fait qu'elle n'exige pas la capacité de production verbale dans la langue cible (langue d'arrivée).

Les partisans de l'intercompréhension le considèrent comme une alternative ou un complément à l'usage courant d'une lingua franca (LF), car une utilisation « non critique » d'une LF peut avoir des conséquences contradictoires (DOYE, 2005; LJOSLAND, 2011), telles que le danger de l'impérialisme linguistique ; la communication insuffisante et la dépréciation potentielle de la langue maternelle ; l'impossibilité d'utiliser une LF sans les idéologies et les pratiques associées à une telle langue.

Loin d'être une idée utopique, des exemples d'utilisation de l'intercompréhension sont mentionnés par la Commission Européenne et soulignent le rôle de l'intercompréhension pour l'intégration européenne: parmi les apprenants des langues romanes (espagnol, portugais, français, italien et roumain); dans le Conseil Nordique et la coopération nordique (Danemark, Finlande, Islande, Norvège et Suède); au Tribunal pénal international de l'exYougoslavie; dans la zone frontalière néerlandaise-allemande; en Suisse ${ }^{13}$. Ces exemples montrent que l'AI peut être une stratégie utile pour les contextes multilingues, au lieu d'adopter une seule lingua franca, comme l'anglais.

Au Brésil, on peut citer des études telles que Henriques (2000), Martins (2014), Carola et Albuquerque (2014) et Ferreira (2018) sur l'intercompréhension. En général, ces travaux montrent le grand potentiel de l'approche

12. Plus d'informations sur: http://coil.suny.edu/

13. Pour plus d'informations sur la Suisse, veuillez vérifier: FINARDI, K.R.; CSILLAGH, V. (2016). Globalization and linguistic diversity in Switzerland: insights from the roles of national languages and English as a foreign language. In: GRUCZA, S.; OLPINSKA-SZKIELKO, M.; ROMANOWSKI, P. (orgs.). Advances in understanding multilingualism: a global perspective. Frankfurt am Main: Peter Lang, pp. 59-79. 
de l'intercompréhension dans l'enseignement et l'apprentissage des langues romanes dans l'enseignement supérieur. Cependant, on peut considérer que la recherche sur cette approche au Brésil est encore à ses débuts et peut être enrichie des propositions que nous présentons dans cet article.

\subsection{Politiques linguistiques}

Les premières études sur les politiques linguistiques (PL) remontent aux années 1950 et 1960, avec les premières interventions dans les nouvelles nations d'Afrique et d'Asie (après leurs indépendances), ainsi que les premières œuvres d'Einar Haugen sur la langue norvégienne (SPOLSKY, 2004; RICENTO, 2006; LAGARES, 2018). Certaines de ces nations devaient adopter le français, comme dans le cas de Congo, Cameroun, Côte d'Ivoire et Senegal. Les PL étaient perçues comme un moyen de résoudre les «problèmes linguistiques » dans les nouvelles sociétés multilingues, en décidant des fonctions que chaque langue aurait dans ces nouveaux pays et en dotant les langues locales d'orthographe, de grammaire et de dictionnaire (LAGARES, 2018).

Les PL peuvent être définies comme l'art de mener des réflexions sur des langues spécifiques, afin de gérer des activités concrètes d'intérêt public, liées aux langues qui comptent pour la population d'un pays, d'un état ou d'instances transnationales plus larges (RAJAGOPALAN, 2013, p. 21, notre traduction) ${ }^{14}$. Les PL existent parce que les langues peuvent être utilisées pour créer une appartenance à un groupe, démontrer l'inclusion/exclusion, montrer le status économique et classer les personnes et les identités personnelles (SHOHAMY, 2006). Les PL traitent également de thèmes liés à la langue étrangère à enseigner / à apprendre, à l'hégémonie d'une langue sur d'autres et aux relations de pouvoir entre les langues d'un même territoire (DAY, 2012).

De plus, les PL existent dans un ensemble complexe de facteurs sociaux, politiques, économiques, religieux, éducatifs et culturels. Spolsky (2004) souligne 3 composantes essentielles des PL: a) pratiques linguistiques - sélection de variétés dans un répertoire linguistique; b) idéologies linguistiques - croyances sur les langues et leur utilisation; c) efforts pour influencer les pratiques - intervention, planification et gestion en langues. Shohamy (2006) ajoute que les PL se produisent au milieu de luttes entre idéologies et pratiques linguistiques, à travers divers mécanismes déclarés et dissimulés, créés par les gouvernements et les grandes entreprises, ainsi que par les systèmes éducatifs.

Wright (2016) aborde les domaines d'intérêt pour les PL: a) la planification du status - le processus juridique consistant à officialiser une langue; b) la planification du corpus - changements linguistiques prévus dans les instituts de langues; c) la planification de l'acquisition - politiques visant à assurer que la langue choisie et planifiée est enseignée et apprise dans les écoles. En bref, les politiques linguistiques sont liées aux structures et processus économiques, politiques et sociaux (et d'autres), dans lesquels les idéologies relatives aux langues affectent le comportement linguistique des utilisateurs de langues (RICENTO, 2006).

Par conséquent, compte tenu de l'importance des politiques linguistiques pour l'enseignement, l'apprentissage et l'utilisation des langues, nous pensons que les PL multilingues et démocratiques peuvent soutenir l'utilisation de plusieurs langues (y compris la langue française) dans les scénarios dominés par l'anglicisation, tels que les universités. Grâce aux efforts et à la participation de différents acteurs du contexte de l'enseignement supérieur, d'autres langues, qui ont été minorisées par la présence de l'anglais, peuvent avoir leur place dans l'internationalisation des universités au Brésil.

\subsection{Politiques linguistiques au Brésil}

La question des politiques linguistiques au Brésil remonte à la période coloniale (DAY, 2012; BAGNO et CARVALHO, 2015). À cette époque, les jésuites ont été expulsés et la langue générale (basée sur le Tupi, une langue autochtone) a été interdite. Le portugais enseigné dans les écoles brésiliennes a (pendant longtemps) été inspiré par la norme littéraire portugaise du XIXe siècle, une sorte de langue très éloignée de la réalité des étudiants brésiliens. Dans les années 1930 et 1940, les variétés de l'allemand et de l'italien étaient interdites pour des raisons idéologiques. Après la Seconde Guerre mondiale, l'émergence des États-Unis en tant que modèle militaire, politique et économique a accru le rôle de la langue anglaise au Brésil, et plusieurs écoles privées ont commencé à proposer

14. Texte original: [...] a arte de conduzir as reflexões em torno de línguas específicas, com o intuito de conduzir ações concretas de interesse público relativo à(s) língua(s) que importam para o povo de uma nação, de um estado ou ainda, instâncias transnacionais maiores. 
l'enseignement de cette langue. Dans le même temps, des pays comme la France et l'Allemagne ont vu leur influence culturelle et économique diminuer, de même que l'intérêt d'apprendre le français et l'allemand (DAY, 2012).

Plus récemment, la Constitution Fédérale (en 1988) indiquait que le portugais était la (seule) langue officielle au Brésil - conformément à l'avis général (et erroné) d'un pays monolingue (LEFFA, 2013). Cependant, l'Inventaire national de la diversité linguistique (INDL) ${ }^{15}$ indique qu'il y a plus de 250 langues au Brésil, y compris les langues autochtones (comme le Tupi-Guarani), les langues ancestrales (comme le Poméranien) et les langues des signes (comme LIBRAS - langue des signes brésilienne).

En 1996, la LDB a indiqué que les écoles (enseignement primaire) pouvaient choisir la langue étrangère à enseigner, en fonction des choix de la communauté locale, compte tenu des ressources disponibles. De manière complémentaire, les Paramètres nationaux du curriculum ( $\mathrm{PCN}$, en portugais) ${ }^{16}$ définissent les critères d'inclusion des langues étrangères dans le curriculum en 1998:

\section{Tableau 1. Critères $d$ 'inclusion des langues étrangères dans le curriculum}

\begin{tabular}{|c|c|}
\hline Type & Description \\
\hline Facteurs historiques & $\begin{array}{l}\text { Liés au rôle que joue une langue donnée à certains moments de l'histoire de l'humanité, de sorte } \\
\text { que l'apprentissage de cette langue devienne pertinent. La pertinence est déterminée par le rôle } \\
\text { hégémonique de ce langage dans les échanges internationaux, notamment dans les domaines de la } \\
\text { culture, de l'éducation, des sciences, du travail, etc. }\end{array}$ \\
\hline $\begin{array}{l}\text { Facteurs liés aux } \\
\text { c o m m u n a u té s } \\
\text { locales }\end{array}$ & $\begin{array}{l}\text { Vivre dans des communautés locales, immigrées ou autochtones peut être un critère pour l'inclusion } \\
\text { d'une langue donnée dans le contexte scolaire. Les justifications de l'inclusion sont: les relations } \\
\text { culturelles, affectives et de parenté. C'est également le cas des communautés linguistiques frontalières. }\end{array}$ \\
\hline $\begin{array}{llll}\text { Facteurs liés à la } \\
\text { tradition }\end{array}$ & $\begin{array}{l}\text { Le rôle que certaines langues étrangères jouent traditionnellement dans les relations culturelles entre } \\
\text { pays peut être un facteur à prendre en compte. Le français, par exemple, a joué et joue un rôle important } \\
\text { du point de vue des échanges culturels entre le Brésil et la France et comme instrument d'accès au savoir } \\
\text { de toute une génération de Brésiliens. }\end{array}$ \\
\hline
\end{tabular}

Source: Adapté des Paramètres nationaux du curriculum (PCN) en 1998 et Day (2012).

Cependant, les changements apportés par la Loi $\mathrm{n}^{\mathrm{0}}$ 13.415/2017 (connue comme la loi de réforme de l'éducation de base) ont eu de profondes répercussions sur l'enseignement et l'apprentissage des langues étrangères au Brésil. À partir de cette année, les écoles doivent proposer l'anglais comme (la seule) langue étrangère obligatoire à enseigner à partir de la 6ème année. Ce sujet sera discuté plus loin dans cette étude.

Dans les lycées, les Paramètres nationaux du curriculum (PCN-EM, en portugais) ${ }^{18}$ indiquent que l'apprentissage d'une langue étrangère implique la prise de conscience de l'acquisition d'un produit culturel complexe. La Loi ${ }^{\circ}$ $11.161 / 2005^{19}$ stipulait que l'espagnol devait être offert dans les écoles secondaires et que les écoles primaires pouvaient proposer cette langue à leurs élèves. Cette décision était liée à la participation du Brésil dans le bloc économique nommé Mercosur (DAY, 2012). A cette époque, cette loi a suscité de vives discussions sur le rôle décroissant d'autres langues dans l'éducation brésilienne, telles que le français et l'italien, par exemple.

Au niveau de l'enseignement supérieur, il n'y a pas de politique linguistique officielle, mais de grands changements ont également eu lieu, grâce aux programmes financés par le gouvernement, tels que les Langues sans Frontières (LsF) et Capes PrInt (PCP), dans le contexte de l'internationalisation de l'enseignement supérieur. Ces programmes ont entraîné la création de politiques linguistiques et de plans d'internationalisation, car les universités membres de ces programmes ont dû créer des politiques et des plans pour pouvoir bénéficier d'un financement du Ministère de l'Education (MEC).

Actuellement, les discussions autour de la Base nationale pour un curriculum commun (BNCC, en portugais) ${ }^{20}$ offrent une occasion de repenser les rôles des langues dans l'éducation. Cette base devrait servir de guide pour les

15. Plus d'informations sur: http://portal.iphan.gov.br/indl

16. Plus d'informations sur: http://portal.mec.gov.br/seb/arquivos/pdf/livro01.pdf

17. Plus d'informations sur: http://www.planalto.gov.br/ccivil_03/_Ato2015-2018/2017/Lei/L13415.htm

18. Plus d'informations sur: http://portal.mec.gov.br/acompanhamento-da-frequeencia-escolar/195-secretarias-112877938/seb-educacaobasica-2007048997/12598-publicacoes-sp-265002211

19. Plus d'informations sur: http://www.planalto.gov.br/ccivil_03/_ato2004-2006/2005/lei/l11161.htm

20. Plus d'informations sur: http://basenacionalcomum.mec.gov.br/ 
programmes des systèmes et réseaux éducatifs des états brésiliens, ainsi que pour les plans pédagogiques de toutes les écoles privées et publiques du Brésil - écoles maternelles, écoles élémentaires et lycées.

\subsection{Le français et autres langues étrangères dans l'internationalisation au Brésil}

Le Brésil est actuellement confronté à une reprise de ses systèmes politique, économique et éducatif. Pays de la taille d'un continent et comptant plus de 209 millions d'habitants, le Brésil possède plus de 250 langues, alors qu'il était la 6ème économie mondiale. La plupart des Brésiliens sont monolingues et ne parlent que la langue officielle, le portugais. Les langues minoritaires telles que le Guarani et les langues ancestrales telles que le Poméranien sont sérieusement menacées au Brésil en raison du manque de politiques linguistiques et éducatives visant à les protéger (FINARDI et GUIMARÃES, 2019).

En ce qui concerne le rôle de la langue française au Brésil, Day (2012) déclare que, à la fin du 19ème siècle, en raison de l'influence culturelle de la France, le français était la langue étrangère principale dans les écoles brésiliennes - la langue française a été introduite dans l'éducation brésilienne entre 1837 et 1857 dans le «Colégio D. Pedro II »(OLIVEIRA, 2015). Les réformes éducatives ultérieures, celles de 1931 (ministre Francisco Campos) et 1942 (ministre Gustavo Capanema) ont mis l'accent sur le développement d'approches didactiques pour l'enseignement des langues étrangères modernes, en particulier du français.

Selon Oliveira (2015), dans les années 1960, la première méthode d'enseignement du français au Brésil était «Voix et Images de France »(VIF), qui a été créée en utilisant la méthodologie SGAV (Structuro-Globale Audio-Visuelle), soutenue par le Ministère de l'Éducation Nationale en France. Les méthodologies actuelles pour enseigner le français impliquent un « apprentissage par tâches », selon les lignes directrices du Cadre européen commun de référence pour les langues (CECRL).

Comme l'ont souligné Finardi (2019b), Leffa (2013) et d'autres, les Brésiliens doivent réfléchir et élaborer des politiques linguistiques qui favorisent la compréhension et la tolérance entre les cultures, les langues et les identités et qui stimulent le multilinguisme et l'égalité des droits et des chances, ainsi qu'un programme d'internationalisation critique et durable (GUIMARÃES, FINARDI et CASOTTI, 2019).

En période de troubles politiques et de conflits de représentation, comme celle que connaît actuellement le Brésil, la réflexion et la promotion de politiques reflétant les souhaits de la population sont encore plus urgentes. Avec la promulgation de la Loi n ${ }^{\circ}$ 13.415/2017 réformant l'enseignement au Brésil, l'anglais est devenu la seule langue étrangère obligatoire dans les écoles, menaçant ainsi l'enseignement / apprentissage d'autres langues étrangères telles que l'espagnol, le français et l'italien.

Par ailleurs, la langue anglaise a l'hégémonie de la production académique dans le monde, dans divers domaines de la connaissance (HAMEL, 2013; FINARDI et FRANÇA, 2016; LAGARES, 2018). En fait, les experts en internationalisation discutent actuellement d'une anglicisation de l'enseignement supérieur (DE WIT, 2011; KNIGHT, 2011b ; FINARDI, 2016b, 2019b) et des possibilités d'inclure dans les cursus des langues autres que l'anglais pour promouvoir le multilinguisme. D'où, la motivation de cette étude.

Selon Finardi (2017), l'utilisation de l'approche d'intercompréhension (IA) et l'offre d'autres langues étrangères telles que l'espagnol, l'italien et le français, pourraient être garanties dans l'enseignement des langues [en général] et dans le contexte de l'internationalisation de l'enseignement supérieur [en particulier] (FINARDI, 2019a). Cependant, les agendas en matière de réformes éducatives et d'internationalisation sont loin d'atteindre un consensus entre les linguistes et les acteurs de l'enseignement supérieur en matière d'utilisation, d'enseignement et de formation.

Par conséquent, il est nécessaire de promouvoir les discussions et de sensibiliser les agents de l'enseignement supérieur à l'importance du multilinguisme dans l'enseignement supérieur, afin que les autres langues ne soient pas minorisées par l'anglais et ne perdent pas leur rôle dans le contexte académique. Afin d'analyser la manière dont la loi générale de l'éducation et des programmes d'internationalisation / langues considèrent le rôle des langues (en général) et du français (en particulier), la section suivante analyse la loi de l'éducation brésilienne, le programme Langues sans Frontières $(\mathrm{LsF})$ et le programme d'internationalisation Capes Print (PCP) et son appel public. 


\section{MATÉRIELS ET MÉTHODES}

Cette étude a utilisé une analyse de la littérature (et recherche documentaire) liée aux langues étrangères et à l'internationalisation pour la comparer aux politiques linguistiques existantes et des textes relatifs aux réformes de l'éducation. Ces sujets sont présentés dans les sous-sections suivantes.

\subsection{Loi des directives nationales pour l'éducation (LDB, en portugais)}

Auteurs tels que Finardi et Archanjo (2015) et Finardi (2016a, 2017) ont révisé la Loi des directives nationales pour l'éducation (LDB, en portugais) qui prévoyait l'offre obligatoire d'une langue étrangère dans le programme d'éducation de base, à partir de la 6ème année et en fonction des besoins/choix de chaque communauté scolaire. Cette loi a été modifiée en 2017 avec la réforme de l'éducation au Brésil et qui, par le biais de la Loi n 13.415/2017, a rendu obligatoire l'offre d'anglais. Des auteurs suggèrent que ces changements dans la loi pourraient avoir des conséquences négatives sur le multilinguisme au Brésil.

D'une part, la loi qui a modifié la LDB en rendant obligatoire l'enseignement de l'anglais semble avoir apporté une solution, au moins partielle, aux problèmes de mise à niveau, de maîtrise de l'anglais et du fossé social causé par l'offre d'une langue dans le secteur privé, tel que décrit par Finardi (2014). En revanche, ce changement de LDB a sérieusement compromis l'offre de langues étrangères autres que l'anglais dans l'enseignement de base (école primaire et secondaire) et, par conséquent, dans l'enseignement supérieur.

Un autre impact sérieux de cette réforme de la loi au Brésil est que les personnes qui étudient actuellement pour devenir professeurs d'espagnol, d'italien et de français (et d'autres langues) peuvent trouver difficile de trouver un emploi après leur diplôme, car les écoles publiques peuvent ne pas leur offrir de possibilités d'emploi.

\subsection{Langues sans Frontières (LsF)}

En ce qui concerne les politiques linguistiques pour l'enseignement supérieur, il n'y a pas de politique explicite pour les langues étrangères au Brésil. Cependant, cela a radicalement changé au cours de la dernière décennie, en raison de l'internationalisation croissante des institutions brésiliennes avec des investissements publics dans des programmes de mobilité tels que Science sans Frontières $(\mathrm{SsF})$, Langues sans Frontières (LsF) et Capes PrInt (PCP). Bien que des programmes aient été supprimés, SsF en 2016 et LsF ${ }^{21}$ en 2019, ils ont affecté les politiques linguistiques dans l'enseignement supérieur en induisant la création de plans d'internationalisation et de politiques linguistiques dans la plupart de leurs établissements membres.

Selon Finardi et Archanjo (2018), le programme LsF peut être considéré comme une politique linguistique implicite et a été créé en raison du manque de compétences (principalement en anglais) des candidats au programme Science sans Frontières (SsF). Le programme Anglais sans Frontières (AsF) a été créé par l'Ordonnance n ${ }^{\circ} 1.466 / 2012$ du Ministère de l'Éducation. Il a été rebaptisé Langues sans Frontières (LsF) par l'Ordonnance no 973/2014 et étendu à de nouveaux publics par l'Ordonnance n ${ }^{\mathrm{O}}$ 30/2016.

Dans toutes les versions du programme, les objectifs reposent sur la formation et l'amélioration linguistique de différentes langues pour la communauté universitaire ou du portugais pour les étrangers à des fins d'internationalisation; sur la formation initiale ou continue de professionnels diplômés en langues étrangères; sur le renforcement de l'enseignement des langues au Brésil; sur l'enseignement de la variété brésilienne de la langue portugaise, ainsi que la culture du pays, à l'étranger; et sur la promotion des politiques linguistiques à des fins d'internationalisation dans le pays.

Le programme comprenait les langues suivantes: allemand, espagnol, français, anglais, italien, japonais et portugais langue étrangère (PLE). En 2019, avant sa suspension par le gouvernement du Brésil, le programme visait à consolider les partenariats, à proposer aux étudiants des tests de compétence, des cours en ligne, et des cours en face à face dans les centres de langues (NucLi, en portugais) installés dans les 96 institutions participantes.

En plus des objectifs susmentionnés, les actions de LsF depuis sa création et son expansion visaient à faciliter et à démocratiser l'accès des étudiants aux principaux tests requis par le monde universitaire international, ainsi qu'à préparer cette communauté à une interaction avec des partenaires internationaux. Le programme LsF avait des

21. Ce programme est mis à jour pour recevoir des fonds autres que ceux du Ministère de l'Éducation. 
activités spécifiques pour la langue française (telles que des cours en ligne et une mise à disposition de conférenciers), en partenariat avec l'Ambassade de France, l'Agence Universitaire de la Francophonie (AUF), Languages Canada et Wallonie Bruxelles.

Au départ le programme comptait avec la participation de 58 universités fédérales. En 2014, il a été élargi pour desservir les 63 universités fédérales de l'époque. En 2017, quatre ans après la création du programme, 58 universités fédérales ont été ré-accréditées et 21 universités d'état, un institut municipal et 16 instituts fédéraux ont été inscrits au programme, totalisant 96 établissements publics accrédités pour le quadriennat 2017-2020.

Les appels publics de réinscription des établissements d'enseignement supérieur pour le programme (appels $\mathrm{n}^{\circ}$ 29, 59 et 100/2017) témoignent des efforts déployés par le programme LsF pour induire la proposition de PL pour l'internationalisation des établissements d'enseignement supérieur participants. Ces appels publics établissaient des critères pour adhérer à l'offre d'autres langues étrangères (en plus de l'anglais) et les institutions ne pouvaient proposer d'autres langues à moins qu'elles s'engagent à offrir le portugais comme langue étrangère/supplémentaire (PLE/PLA, en portugais).

Les établissements d'enseignement supérieur avaient la possibilité d'offrir uniquement l'anglais (offre de type 1), l'anglais et le PLE (offre de type 2) et l'anglais, le PLE et d'autres langues (offre de type 3). Afin d'encourager le multilinguisme et la gamme la plus large possible, la ressource de chaque établissement d'enseignement supérieur serait adaptée au modèle d'offre adopté. Une autre preuve du caractère inductif du PL pour l'internationalisation des décrets d'accréditation LsF est le fait que cette annonce demandait l'officialisation d'un PL institutionnel pour adhérer à l'annonce. Le Tableau 2 ci-dessous présente l'offre linguistique dans le programme avant son interruption.

Tableau 2. Langues proposées par catégories d'établissements accrédités par LsF

\begin{tabular}{|l|l|l|l|l|l|l|l|}
\hline Type & Anglais & PLE/PLA & Français & Espagnol & Allemand & Italien & Japonais \\
\hline Universités Fédérales & 58 & 50 & 34 & 32 & 14 & 10 & 5 \\
\hline Universités D'état / Municipales & 22 & 10 & 2 & 4 & 5 & 3 & 1 \\
\hline Instituts Fédéraux & 16 & 0 & 0 & 0 & 0 & 0 & 0 \\
\hline Total & 96 & 60 & 36 & 36 & 19 & 13 & 6 \\
\hline
\end{tabular}

Source: De Abreu-e-Lima et Finardi (2019)

On peut voir que le français a joué un rôle important dans l'offre de langues étrangères dans ce programme, avec l'espagnol, bien qu'un nombre plus élevé d'institutions aient proposé l'anglais. Cela peut indiquer le rôle croissant du français dans le contexte de l'internationalisation des universités brésiliennes.

\subsection{Programme Capes PrInt (PCP)}

Le programme Capes Print (PCP) a été créé en 2017 (et différemment de SsF, il se concentre sur le niveau post-universitaire) dans le but de:

a. favoriser la construction, la mise en œuvre et la consolidation des plans d'internationalisation stratégiques des institutions participantes dans les domaines de connaissance privilégiés;

b. encourager la formation de réseaux de recherche internationaux pour améliorer la qualité de la production universitaire liée aux études de troisième cycle;

c. développer les actions de soutien à l'internationalisation chez les diplômés des institutions envisagées;

d. promouvoir la mobilité des enseignants et des étudiants, en particulier des doctorants, des post-doctorants et des professeurs à l'étranger et au Brésil, en liaison avec des programmes d'études supérieures stricto-sensu avec coopération internationale;

e. encourager la transformation des institutions participantes dans un environnement international ${ }_{i}$

f. intégrer d'autres actions de promotion de Capes à l'effort d'internationalisation.

L'appel public de Capes Print a incité les universités à approuver une politique ou un plan d'internationalisation institutionnelle, afin de soumettre leurs propositions de financement pour ce programme. Donc, le PCP représente une preuve de la politique linguistique implicite de la Capes, parce que les propositions pour l'appel public PCP doivent être faites en anglais. 
Une autre indication de la promotion de la langue anglaise est la liste ${ }^{22}$ des pays prioritaires pour la coopération dans ce programme. La plupart des pays utilisent l'anglais comme langue première ou supplémentaire. Par conséquent, des efforts pourraient être faits pour promouvoir la langue française dans la coopération (au lieu de l'anglais comme lingua franca) avec des pays tels que la Belgique, le Canada, la France et la Suisse.

\section{DISCUSSION}

La revue de la littérature, en comparaison avec les documents gouvernementaux existants relatifs à l'éducation (en général) et à l'éducation linguistique (en particulier), indique que l'internationalisation et les politiques linguistiques sont des phénomènes imbriqués. Au Brésil, la création de politiques linguistiques et de plans d'internationalisation semble être motivée par des programmes financés par le gouvernement fédéral, tels que SsF, LsF et PCP. De plus, les changements dans l'enseignement primaire et secondaire (liés à l'enseignement des langues étrangères) ont eu des impacts dans l'enseignement supérieur.

Le choix des langues étrangères à utiliser dans l'internationalisation (principalement l'anglais) semble également être influencé par ces programmes et par les tendances internationales en matière d'anglicisation de l'enseignement supérieur, comme l'on a discuté précédemment dans cette étude - laissant de côté l'importance historique du français dans l'éducation brésilienne, par exemple. Ces choix se produisent de manière descendante (top-down), ne laissant que peu de place aux discussions ascendantes (bottom-up) qui pourraient prendre en compte les besoins des institutions locales et les efforts pour promouvoir les environnements multilingues.

Cela nous amène à penser comment les choix en matière de langues dépendent tellement des décisions gouvernementales. Avec le financement décroissant de l'enseignement public, il faut penser à des alternatives pour promouvoir les langues autres que l'anglais. C'est pourquoi l'approche d'intercompréhension peut représenter une option importante pour les contextes où d'autres langues (comme le français) sont minorisées par l'anglais - en utilisant cette approche, on peut faire face aux défis posés par les changements de législation qui affectent l'utilisation des langues.

\section{CONCLUSION}

Cette étude a débuté par une discussion sur les impacts de la mondialisation sur l'éducation (en général) et sur l'internationalisation de l'enseignement supérieur (en particulier). Après cela, la revue de littérature a abordé des thèmes importants tels que l'internationalisation, l'intercompréhension, les politiques linguistiques (en général), les politiques linguistiques au Brésil et le rôle du français et d'autres langues pour l'internationalisation au Brésil.

On peut conclure que les plans d'internationalisation et les politiques linguistiques en vigueur dans l'enseignement supérieur tendent à favoriser l'anglais au détriment d'autres langues pertinentes, telles que le français, l'espagnol et l'italien, par exemple. Par conséquent, des alternatives sont nécessaires pour promouvoir le multilinguisme dans l'enseignement supérieur, et l'une de ces alternatives est l'approche de l'intercompréhension.

Il faut rappeler que, dans le cas du français, qui a joué un rôle important dans l'histoire de l'éducation brésilienne, l'intercompréhension semble être une alternative intéressante, puisque le français et le portugais appartiennent à la même «famille » de langues (partageant de nombreuses similitudes). Avec une vision démocratique et inclusive de l'utilisation des langues dans l'enseignement supérieur au Brésil, les membres des universités pourraient déplacer leur attention de l'anglais vers d'autres langues, en particulier le français, qui avait et a un rôle si important dans divers domaines de la recherche et d'enseignement de nos jours.

On peut aussi remarquer que, compte tenu de la diminution des financements pour l'enseignement supérieur public (en particulier la diminution des ressources destinées à la mobilité universitaire et à la recherche internationale), l'utilisation des technologies de l'information et de la communication (TICs) est fondamentale pour le développement de stratégies telles que «l'internalisation chez soi » $(\mathrm{IaH})$ et la mobilité académique virtuelle comme la COIL. De plus, chercher des alternatives est également un besoin urgent pour le travail du linguiste appliqué, car les politiques

22. Plus d'informations sur: https://www.capes.gov.br/images/stories/download/editais/02022018-Edital-41-2017-Print-alteracao-anexo-1.pdf 
actuelles projettent un avenir incertain pour le travail des professeurs de langues qui n'enseignent pas les langues hégémoniques, comme dans le cas de l'anglais.

\section{RÉFÉRENCES BIBLIOGRAPHIQUES}

ALTBACH, P.G.; KNIGHT, J. (2007). The internationalization of higher education: motivations and realities. Journal of Studies in International Education, v. 11, pp. 290-305.

ALTENHOFEN, C.V. (2013). Bases para uma política linguística das línguas minoritárias no Brasil. In: Nicolaides, C. et al. (orgs.). Política e Políticas Linguísticas. Campinas: Pontes Editores, pp. 93-116.

ARAÚJO E SÁ, M.H.; SIMÕES, A.R. (2015). Integración curricular de la Intercomprensión: posibilidades, limitaciones, recomendaciones (relatório). Brussels: European Union.

BAGNO, M.; CARVALHO, O.L.S. (2015). O potencial do português brasileiro como língua internacional. Revista Interdisciplinar, v. 22, pp. 11-26.

BEELEN, J.; JONES, E. (2015). Redefining Internationalization at Home. In: Curaj, A. et al. (orgs.). The European Higher Education Area: between critical reflections and future policies. Cham: Springer, pp. 59-72.

BERNHEIM, C.T. (2008). La educación superior en América Latina y el Caribe: diez años después de la Conferencia Mundial de 1998. Bogotá: UNESCO.

BLOMMAERT, J. (2013). Citizenship, language and superdiversity: towards complexity. Journal of Language, Identity and Education, v. 12, pp. 193-196.

BRUTT-GRIFFLER, J. (2017). English in the multilingual classroom: implications for research, policy and practice. PSU Research Review, v. 1, n. 3, pp. 216-228.

CANAGARAJAH, S. (2013). Navigating language politics: a story of critical praxis. In: Nicolaides, C. et al. (orgs.). Politica e Políticas Linguísticas. Campinas: Pontes Editores, pp. 43-61.

CAROLA, C.; ALBUQUERQUE COSTA, H. (2014). Intercompreensão no ensino de línguas estrangeiras: formação plurilíngue para pré-universitários. Revista Moara, v. 42, pp. 99-116.

DAY, K. (2012). Ensino de língua estrangeira no Brasil: entre a escolha obrigatória e a obrigatoriedade voluntária. Revista Escrita, v. 15, pp. 1-13.

DE ABREU-E-LIMA, D.P.M.; FINARDI, K.R. (2019). Políticas linguísticas para internacionalização e o papel do programa Idiomas sem Fronteiras. In: Finardi, K.; Scherre, M.M.; Vidon, L. (orgs.). Língua, Discurso e Política: desafios contemporâneos. Campinas: Pontes Editores, pp. 13-28.

DE BIASE, A. (2013). Intercomprébension: analyse des stratégies d'apprentissage pour comprendre les langues romanes. Dissertação de Mestrado. Haute École Pédagogique, Lausanne.

DE OLIVEIRA, J.M.F. (2016). A intercompreensão de línguas românicas nas aulas de inglês: uma experiência inovadora nos cursos de Educação de Jovens e Adultos (EJA) do Instituto Federal do Rio Grande do Norte (IFRN). Dissertação de Mestrado. Universidade Federal do Rio Grande do Norte, Natal.

DE SOUSA SANTOS, B. (2011). Epistomologías del Sur. Utopía y Praxis Latinoamericana, v. 16, n. 54, pp. $17-39$.

DE WIT, H. (2011) Internationalization of Higher Education: nine misconceptions. International Higher Education, v. 64, pp. 6-7.

DE WIT, H.; HUNTER, F.; HOWARD, L.; EGRON-POLAK, E. (2015). Internationalisation of Higher Education (relatório). Brussels: European Parliament. 
DOYÉ, P. (2005). Intercomprebension: guide for the development of language education policies in Europe - from linguistic diversity to plurilingual education (relatório). Strasbourg: Council of Europe.

EUROPEAN COMMISSION (2012). Studies on translation and multilingualism: Intercomprehension. Brussels: European Union.

FERREIRA, L.G. (2018). Política Linguística: a bistoriografia da oferta de línguas estrangeiras no Brasil e a intercompreensão como ferramenta de valorização do plurilinguismo. Trabalho de conclusão de curso de pós-graduação em Letras, Universidade Federal do Paraná, Curitiba.

FINARDI, K.R. (2014). The slaughter of Kachru's five sacred cows in Brazil: affordances of the use of English as an international language. Studies in English Language Teaching, v. 2, pp. 401-411.

FINARDI, K.R. (2016a). Language policies and internationalisation in Brazil: the role(s) of English as an additional language. In: Sciriha, L. (org.). International Perspectives on Bilingualism. Newcastle upon Tyne: Cambridge Scholars Publishing, pp. 79-90.

FINARDI, K.R. (2016b). English in Brazil: views, policies and programs. Londrina: EDUEL.

FINARDI, K.R. (2017). What Brazil can learn from multilingual Switzerland and its use of English as a multilingua franca? Acta Scientiarum, v. 39, pp. 219-228.

FINARDI, K.R. (2019a). Internationalization and multilingualism in Brazil: possibilities of Content and Language Integrated Learning and Intercomprehension approaches. International Journal of Educational and Pedagogical Sciences, v. 13, pp. 656-659.

FINARDI, K.R. (2019b). English in the South. Londrina: EDUEL.

FINARDI, K.R.; ARCHANJO, R. (2018). Washback effects of the Science without Borders, English without Borders and Language without Borders programs in Brazilian language policies and rights. In: Siiner, M.; Hult, F.; Kupisch, T. (orgs.). Language Policy and Language Acquisition Planning. Cham: Springer, pp. 173-185.

FINARDI, K.R.; FRANÇA, C. (2016). O Inglês na internacionalização da produção científica brasileira: evidências da subárea de linguagem e linguística. Revista Intersecções, v. 19, pp. 234-250.

FINARDI, K.R.; GUIMARÃES, F.F. (2019). Local agency in national language policies: the internationalisation of higher education in a Brazilian institution. Current Issues in Language Planning, v. 20, n. 5, pp. 1-23.

FINARDI, K.R.; PORCINO, M.C. (2014). Tecnologia e metodologia no ensino de inglês: impactos da globalização e da internacionalização. Revista Itha do Desterro, v. 66, pp. 239-282.

FINARDI, K.R.; ROJO, R.A.O. (2015). Globalization, internationalization and education: what is the connection? International e-Journal of Advances in Education, v. 1, n. 1, pp. 18-25.

FINARDI, K.R.; ARCHANJO, R. (2015). Reflections of internationalization of education in Brazil. In: International Business and Education Conference 2015 Proceedings. New York: Clute Institute, pp. 504-510

GUIMARÃES, F.F.; AMORIM, G.B.; PICCIN, G.F.O.; FINARDI, K.R.; DE SOUZA, N. (2019). Intercompreensão e Internacionalização: construindo uma cidadania glocal sustentável. In: Finardi, K. et al. (orgs.). A diversidade de fazeres em torno da linguagem: universidades, faculdades e educação básica em ação. Campinas: Pontes Editores, pp. 217-230.

GUIMARÃES, F.F.; FINARDI, K.R.; CASOTTI, J.B.C. (2019). Internationalization and language policies in Brazil: what is the relationship? Revista Brasileira de Linguística Aplicada, v. 19, n. 2, pp. 295-327.

GUIMARÃES, F.F.; FINARDI, K.R.; EL KADRI, M.S.; TAQUINI, R. (2020). The mission statements of the federal universities and the projection of internationalization in Brazil. System, v. 94, pp. 1-13.

GUIMARÃES, F.F.; KREMER, M.M. (2020). Adopting English as a medium of instruction (EMI) in Brazil and Flanders (Belgium): a comparative study. Revista Ilha do Desterro, v. 73, n. 1, pp. 217-246. 
HAMEL, R.E. (2013). El campo de las ciencias y la educación superior entre el monopolio del inglés y el plurilingüismo: elementos para una política del lenguaje en América Latina. Trabalbos em Linguística Aplicada, v. 52, n. 2, pp. 321-384.

HENRIQUES, E.R. (2000). Intercompreensão de texto escrito por falantes nativos de português e de espanhol. Revista D.E.L.T.A., v. 16, n. 2, pp. 263-295.

HILDEBLANDO JÚNIOR, C.A.; FINARDI, K.R. (2018). Internationalization and virtual collaboration: insights from COIL experiences. Revista Ensino em Foco, v. 1, n. 2, pp. 19-33.

HUDZIK, J. (2011). Comprehensive Internationalization: from concept to action. Washington D.C.: NAFSA, 2011.

JENKINS, J. (2015). Repositioning English and multilingualism in English as a lingua franca. Englishes in Practice, v. 2, n. 3, pp. $49-85$.

JORDÃO, C.M. (2016) Decolonizing identities: English for internationalization in a Brazilian university. Interfaces - Brasil / Canada, v. 16, n. 1, pp. 191-209.

KNIGHT, J. (2003). Updating the definition of internationalization. International Higher Education, v. 33, pp. 2-3.

KNIGHT, J. (2011a). Five myths about internationalization. International Higher Education, v. 62, pp. $14-15$.

KNIGHT, J. (2011b) Doubts and dilemmas with double degree programs. Revista de Universidad y Sociedad del Conocimiento, v. 8, n. 2, pp. 297-312.

KNOBEL, M. (2012). Brazil seeks academic boost by sending students abroad. International Higher Education, v. 66, pp. 147-149.

LAGARES, X.C. (2018). Qual política linguística? Desafios glotopolíticos contemporâneos. São Paulo: Parábola Editorial.

LAUS, S.P. (2012). A internacionalização da educação superior: um estudo de caso da Universidade Federal de Santa Catarina. Tese de Doutorado. Universidade Federal da Bahia, Salvador.

LEFFA, V.J. (2013). Prefácio. In: Nicolaides, C. et al. (orgs.). Política e Políticas Linguísticas. Campinas: Pontes Editores, pp. 7-10.

LJOSLAND, R. (2011). English as an academic lingua franca: language policies and multilingual practices in a Norwegian university. Journal of Pragmatics, v. 43, n. 4, pp. 991-1004.

MANÇOS, G.R.; COELHO, F.S. (2017). Internacionalização da ciência brasileira: subsídios para avaliação do programa Ciência sem Fronteiras. Revista Brasileira de Políticas Públicas e Internacionais, v. 2, n. 2, pp. 52-82.

MARTINS, S.A. (2014). A intercompreensão de línguas românicas: proposta propulsora de uma educação plurilíngue. Revista Moara, v. 42, pp. 117-126.

MEISSNER, F. J. (2010). La didáctica de la Intercomprensión y sus repercusiones en la enseñanza de lenguas: el ejemplo alemán. Synergies Chili, v. 6, pp. 59-70.

MENEGHEL, S.M.; DE CAMARGO, M.S.; SPELLER, P. (2018). De Havana a Córdoba: duas décadas de educação superior na América Latina. Brasília: Editora Novaletra.

OLIVEIRA, R. S. (2015). Linha do tempo da didática das línguas estrangeiras no Brasil. Non Plus, v. 7, pp. 27-38.

RAJAGOPALAN, K. (2013). Política Linguística: do que é que se trata, afinal? In: Nicolaides, C. et al. (orgs.) Política e Políticas Linguísticas. Campinas: Pontes Editores, pp. 19-42.

RAMOS-GARCÍA, A.M.; VÁZQUEZ, V.P. (2018). The linguistic internationalization of higher education: a study on the presence of language policies and bilingual studies in Spanish universities. Porta Linguarum, Monográfico III, pp. 31-46.

RICENTO, T. (2006). An introduction to Language Policy: theory and method. Malden, MA: Blackwell Publishing.

SHOHAMY, E. (2006). Language Policy: bidden agendas and new approaches. New York: Routledge. 
SPOLSKY, B. (2004). Language Policy. New York: Cambridge University Press.

STEIN, S.; ANDREOTTI, V.O. (2017). Afterword: provisional pedagogies toward imagining global mobilities otherwise. Curriculum Inquiry, v. 47, n. 1, pp. 135-146.

VERTOVEC, S. (2007). Super-diversity and its implications. Ethnic and Racial Studies, v. 30, n. 6, pp. 1024-1054.

WANG, X.; SPOTTI, M.; JUFFERMANS, K.; CORNIPS, L.; KROON, S.; BLOMMAERT, J. (2014). Globalization in the margins: toward a re-evaluation of language and mobility. Applied Linguistics Review, v. 5, n. 1, pp. 23-44.

WRIGHT, S. (2016). Language policy and planning: from nationalism to globalization. New York: Palgrave Macmillan.

Recebido : 18/7/2020

Aceito : 3/11/2020

Publicado : 2/3/2021 